A. Weisbecker, M. Burmester \& A. Schmidt (Hrsg.): Mensch und Computer 2015 Workshopband, Stuttgart: Oldenbourg Wissenschaftsverlag, 2015, S. 165-169.

\title{
Gestaltung gebrauchstauglicher Assistenzsysteme für Industrie 4.0
}

\author{
Michael Wächter, Angelika C. Bullinger \\ Professur Arbeitswissenschaft und Innovationsmanagement, TU Chemnitz
}

\section{Zusammenfassung}

Die Entwicklung mobiler Assistenzsysteme für die Instandhaltung stellt hinsichtlich der späteren Akzeptanz durch den Anwender eine große Herausforderung dar. Dieser Artikel stellt ein Konzept zur kollaborativen Entwicklung eines mobilen Assistenzsystems für Instandhalter vor. Die Anwender erhalten dabei durch die Verwendung von Hardware-Prototyping schon in frühen Phasen der Produktgestaltung die Möglichkeit, Kriterien der Gebrauchstauglichkeit zu bewerten und mit zu gestalten. Unter Verwendung verschiedener Methoden der Produktergonomie und Designtechnik werden die einzelnen Phasen der Produktentwicklung von der Anforderungsanalyse bis zum Funktionsprototyp konzeptionell aufgezeigt.

\section{Einleitung}

Industrie 4.0 steht für die intelligente Vernetzung der gesamten Wertschöpfungskette. Cyberphysische Produktionssysteme stimmen ihre Aufträge, benötigtes Material und Informationen in Selbstorganisation untereinander ab und koordinieren mit Hilfe permanent verfügbaren Statusinformationen Inspektionen, Wartungen und Instandhaltungsmaßnahmen (BITKOM 2014; acatech 2014). Die kontinuierlich bereitgestellten Informationen der Maschinen und Anlagen sollen genutzt werden, um neben der Bereitstellung anwenderspezifischer Informationen auch den Produktionsprozess zu optimieren (Bilek et al. 2012; BMWi 2014). Instandhalter sollen zukünftig z.B. über ein mobiles Assistenzsystem alle relevanten Informationen, Dokumentationen und Anleitungen erhalten. Dies dient in erster Linie der effizienten Durchführung von Instandhaltungsmaßnahmen (Scheer 2013). Aktuell beschränkt sich der Einsatz von mobilen Endgeräten im Produktionsbereich auf wenige Anwendungsfälle, wird aber künftig in Folge der großen Datenverfügbarkeit an Bedeutung gewinnen (Fraunhofer IAO 2014). Im Mittelpunkt der Entwicklung stehen dabei die Gestaltung der Benutzeroberfläche (Software-Usability) und einer geeigneten Hardware (Hardware-Usability). Während in der Softwareentwicklung eine Vielzahl an Methoden und Leitfäden zur Gestaltung gebrauchstauglicher Oberflächen existieren, besteht im Bereich der Hardware-Usability noch 
kaum genutztes Potential bei der kontextspezifischen Mensch-Computer Interaktion (Gerhard 2009).

Die in diesem Beitrag vorgestellte Konzeptidee zeigt zur Schließung dieser Lücke auf, wie die Hardware eines mobilen Assistenzsystems für Instandhalter kollaborativ mit zukünftigen Anwendern entwickelt werden kann.

\section{Methodische Vorgehensweise}

Um die Anforderungen des Instandhaltungspersonals an ein mobiles Assistenzsystem zu erforschen, wurde eine detaillierte Anforderungs- und Nutzerkontextanalyse in Unternehmen der Automobilindustrie, der Automobil-Zulieferindustrie sowie der Windkraftbranche durchgeführt. Mittels Expertenbefragungen, Prozessbeobachtungen und der Erstellung von Use-Cases wurden die Anforderungen an Dateninhalte, -komplexität sowie Hard- und Software erfasst. In den folgenden Betrachtungen liegt der Fokus auf den Anforderungen der Anwender an die Hardware eines mobilen Assistenzsystems und der methodischen Vorgehensweise bei der ergonomischen Gestaltung der Hardwareelemente.

\subsection{Anforderungsanalyse}

Trotz der steigenden Verfügbarkeit verschiedener mobiler Endgeräte wie Datenbrillen, Smartphones oder Smartwatches, halten die mittels leitfadengestützten Experteninterview befragten Instandhalter $(n=18)$ den Einsatz eines Tablets mit einer Displaydiagonalen von acht bis zehn Zoll zur Anzeige von Dateninhalten wie z.B. Zeichnungen für effektiv. Die Möglichkeit zur Eingabe von Informationen über eine physische Tastatur, die Robustheit für den industriellen Einsatz und eine einfache, intuitive Bedienung stellen die nichtfunktionalen Anforderungen als Ergebnis der Anforderungsanalyse dar. Im Zuge der Nutzerkontextanalyse ergaben sich durch die Betrachtung verschiedener Use-Cases folgende funktionale Anforderungen:

- Transportmöglichkeit

- Bedienung mittels Touchscreen und physischer Tasten, Joystick oder Touchpad

- Funktion zum Hinstellen auf ebenen Flächen

- Funktion zum Anheften an Maschinen und Anlagen während der Montage

Die ermittelten funktionalen und nichtfunktionalen Anforderungen bilden die Basis für die hardwaretechnische Entwicklung des mobilen Assistenzsystems.

\subsection{Rapid Prototyping}

Um die Anwender schon zu einem frühen Zeitpunkt in die Produktentwicklung einbeziehen zu können, eignet sich die Anfertigung von hardwaretechnischen Prototypen. Die späteren 
Nutzer erhalten so die Möglichkeit, die gewünschten Anforderungen an die Funktion des Produktes aber auch an die ergonomische Gestaltung, die Haptik und die Optik zu bewerten und dem Entwickler schon in den frühen Phasen der Produktentstehung Feedback hinsichtlich der subjektiv wahrgenommenen Usability zurück zu spiegeln. Rapid Prototyping verfolgt das Ziel, ein möglichst realitätsnahes, greifbares Abbild des Produktes zur Verfügung zu stellen, das die Eigenschaften so gut wie möglich darstellt (Bertsche \& Bullinger 2007).

Prototypen werden hinsichtlich ihres Umfanges unterschieden. Ein Konzeptmodell umfasst die Form und Beschaffenheit der Oberflächen und beinhaltet keine mechanischen Eigenschaften des Endproduktes. Im Geometrieprototyp nähert sich der Entwickler der definierten Produktform exakt an und kann anschließend die Ergonomie und Haptik des Produktes testen sowie evaluieren. Ein Funktionsprototyp repräsentiert den Großteil der späteren Produkteigenschaften und dient Versuchen in der späteren Anwendungsumgebung (Bonten 2003).

\subsection{Ideation, Design und Prototyping}

Zur Erstellung eines Konzeptmodells wird zunächst eine Fokusgruppe mit mehreren Probanden durchgeführt. Um in dieser Phase möglichst viele Ideen zu generieren, können auch Nicht-Anwender an der Fokusgruppe teilnehmen. Dafür werden die Teilnehmer einer praktischen Übung zum nutzerzentrierten Entwicklungsprozess in fünf Gruppen mit mindestens drei Personen eingeteilt. Um die Ausgangsituation zur Gestaltung der Konzeptmodelle zu vereinheitlichen, erhalten die Probanden als Grundlage die funktionalen und nichtfunktionalen Produkteigenschaften aus der Anforderungs- und Nutzerkontextanalyse. Die Phase der Ideenfindung verwendet die Methoden des Design-Thinking (Meinel et al. 2009). Die Ideengenerierung erfolgt dabei mittels Brainstorming. Anschließend haben die einzelnen Gruppen die Möglichkeit, einen Designentwurf mit Hilfe von Modelliermasse anzufertigen und vor den anderen Gruppen zu präsentieren. Am Ende dieser Phase stehen somit fünf Konzeptmodelle zur Verfügung, die in einem Workshop mit Experten der Produktergonomie hinsichtlich der Erfüllung der Usability-Kriterien bewertet und in ein einheitliches Konzeptmodell übertragen werden.

Dieses Konzeptmodell wird anschließend mittels 3D-Scanner digitalisiert und dient als Grundlage für den Geometrieprototyp. Unter Verwendung digitaler Menschmodelle erfolgt eine Untersuchung des Konzeptmodells hinsichtlich anthropometrischer Variablen. Dabei werden die Greifräume und Greifarten des 5. Perzentil der Frau und 95. Perzentil des Mannes nach DIN 33402 (2005) analysiert und in einen primären, sekundären und tertiären Greifraum sowie den Kontaktbereich unterteilt. Mit Hilfe dieser Analyse können die Maße der Griffe und die Anordnung der Tasten, Joysticks und anderer physischer Bedienelemente sowie die Erreichbarkeit des Touchscreens angepasst und weiterentwickelt werden. Im nächsten Schritt wird der erarbeitete Geometrieprototyp mittels FDM - Verfahren gedruckt und in einem Workshop mit den späteren Anwendern diskutiert und bewertet. Diese Phase wird nach Bedarf iterativ durchgeführt.

Nach der erfolgreichen Gestaltung des Konzeptmodells erfolgt die Entwicklung eines Funktionsprototyps. Während der Touchscreen bis zu diesem Stadium nur durch eine Plexiglas- 
platte angedeutet wird, ist dieses Bedienelement inklusive einer Testanwendung im Funktionsprototyp verbaut. Mit Hilfe des Funktionsprototyps erfolgt die Evaluation des entwickelten mobilen Assistenzsystems anhand der aufgenommenen Use-Cases.

\section{Diskussion und Ausblick}

Die hier beschriebene Konzeptidee folgt dem Forschungsansatz des Design Science Research. Dabei bedient sich die Methodik an bestehenden Modellen (User-Centered Design, Participatory Design, Design Thinking) und adaptiert diese an die Anforderungen der Produktentwicklung, speziell im Bereich Hardware. Der Einsatz von digitalen Menschmodellen zur Berücksichtigung anthropometrischer Faktoren und die digitale Modellierung der Konzeptmodelle sichert schon in den frühen Phasen der Entwicklung eine hohe Qualität der Prototypen. Durch die iterative Anpassung der Geometrieprototypen im Austausch mit den Anwendern lassen sich Anregungen und Verbesserungen sofort umsetzen und zurückspiegeln. Die gewonnen Erkenntnisse bilden eine solide Basis für die Umsetzung des Funktionsprototyps und die erfolgreiche Evaluation. Die kontinuierliche Einbeziehung der Anwender soll die Akzeptanz sichern zu einem hohen Nutzungsgrad beitragen.

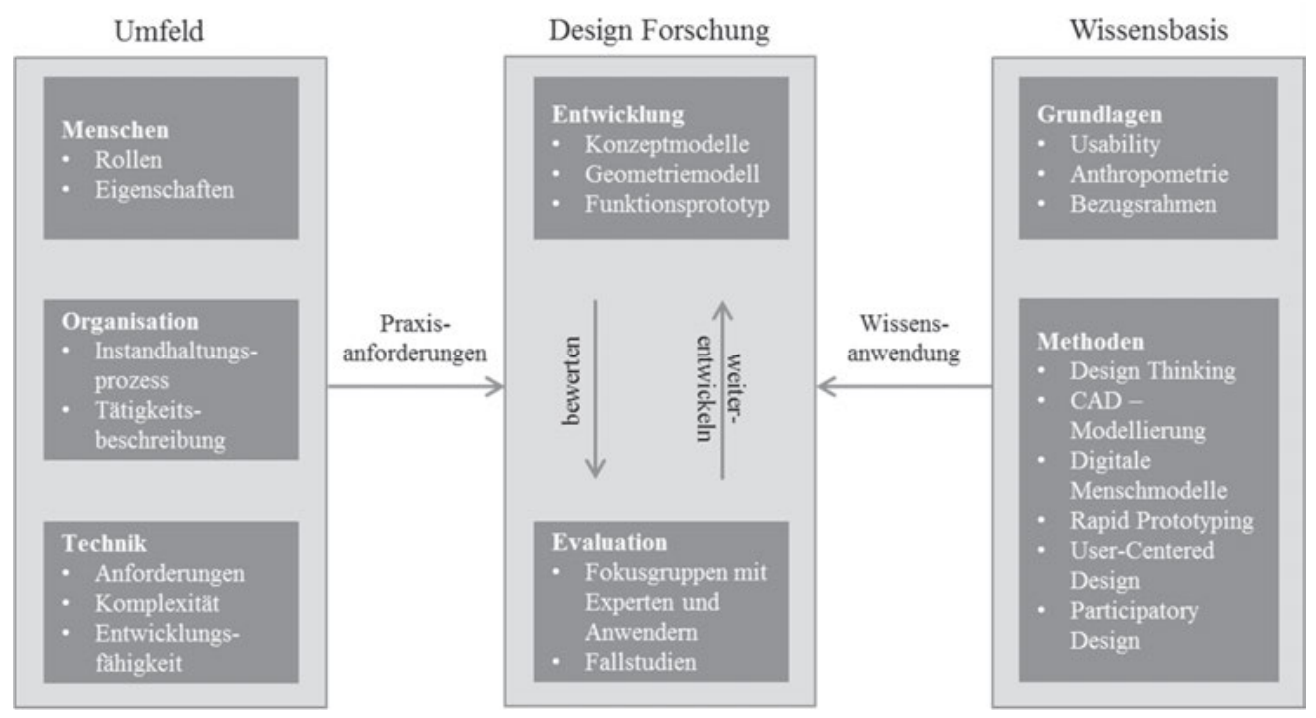

Abbildung 1: Methodischer Forschungsansatz nach Hevner et al. (2004)

Die vorgestellte Vorgehensweise ist Teil eines Promotionsvorhabens und soll nach erfolgreicher Entwicklung des mobilen Assistenzsystems für Instandhalter in eine Methodenbeschreibung überführt werden. 


\section{Danksagung}

Dieser Beitrag entstand im Rahmen des Projekts „Ressourcen-Cockpit für Sozio-CyberPhysische Systeme“. Dieses Forschungs- und Entwicklungsprojekt wird mit Mitteln des Bundesministeriums für Bildung und Forschung (BMBF) im Rahmenkonzept „Forschung für die Produktion von morgen“ gefördert und vom Projektträger Karlsruhe (PTKA) betreut. Die Verantwortung für den Inhalt dieser Veröffentlichung liegt bei den Autoren.

\section{Literaturverzeichnis}

Acatech (2012). agendaCPS: Integrierte Forschungsagenda Cyber-Physical Systems. Geisberger, E.; Broy, M. (Hrsg.). acatech STUDIE.

Bilek, E., Busch, F., Hartung, J., Scheele, C., Thomas. C., Deuse, J. \& Kuhlenkötter, B. (2012). Intelligente Erstellung und Nutzung von Maschinendokumentation. ZWF Zeitschrift für wirtschaftlichen Fabrikbetrieb 107, S. 652-656.

Bertsche, B. \& Bullinger, H. J. (2007). Entwicklung und Erprobung innovativer Produkte-Rapid Prototyping. Springer-Verlag Berlin Heidelberg.

BITKOM (2014). Industrie 4.0 - Volkswirtschaftliches Potenzial für Deutschland. Fraunhofer IAO (Hrsg.).

BMWi (2014). Zukunft der Arbeit in Industrie 4.0. Bundesministerium für Wirtschaft und Energie (Hrsg.).

Bonten, C. (2003). Kunststofftechnik für Designer. München, Wien: Carl Hanser Verlag.

DIN 33402 (2005). Ergonomie - Körpermaße des Menschen. Beuth, Berlin.

DIN EN ISO 9241-210 (2011). Ergonomie der Mensch-System-Interaktion-Teil 210: Prozess zur Gestaltung gebrauchstauglicher interaktiver Systeme. Beuth, Berlin.

Fraunhofer IAO (2014). Produktionsarbeit der Zukunft - Industrie 4.0. Fraunhofer Verlag.

Gerhard, M. (2009). Akzeptanz mobiler räumlicher Assistenzsysteme. In Großmann, U. (Hrsg.): Innovative mobile Technologien., Berlin: LIT Verlag, S. 39-50.

Hevner, A. R., March, S. T., Park, J., Ram, S. (2004). Design Science in Information Systems Research. MIS Quarterly 28, S. 75-105.

Meinel, H., Weinberg, C. \& Plattner, U. (2009). Design Thinking. Hasso Plattner Institut.

Scheer, A.-W. (2013). Industrie 4.0 - Wie sehen Produktionsprozesse im Jahr 2020 aus?. IMC AG.

\section{Kontaktinformationen}

Michael Wächter

Technische Universität Chemnitz

09107 Chemnitz

E-mail: michael.waechter@mb.tu-chemnitz.de 
